\title{
On-Capillary Raman Spectroscopy of Nucleoside / Borate Complexes Separated by Isotachophoresis
}

\author{
Hongmin Li, Patrick A. Walker III, Michael D. Morris \\ University of Michigan, Department of Chemistry, 930 N. University, Ann Arbor, MI 48109-1055, USA
}

Received 28 July 1997; accepted 30 October 1997

\begin{abstract}
On-capillary normal Raman spectroscopy with $532 \mathrm{~nm}$ excitation is used as a detection method for capillary isotachophoresis (ITP) of the nucleosides: adenosine, cytidine, guanosine, and uridine. The compounds are separated as their borate complexes between leading electrolyte $0.1 \mathrm{M} \mathrm{Cl}^{-}, \mathrm{pH} 7.5$, and terminating electrolyte $0.1 \mathrm{M} \mathrm{B}(\mathrm{OH})_{4}^{-}, \mathrm{pH} 10$. The Raman spectra of the nucleoside/borate complexes are presented. Additionally, Raman spectra are obtained from separated adenosine and adenosine $5^{\prime}$-triphosphate (ATP). Starting concentrations in the range $5.0 \times 10^{-6}-5 \times 10^{-4} \mathrm{M}$ are employed. Preconcentration factors of at least $10^{3}$ are observed. (c) 1998 John Wiley \& Sons, Inc. J Micro Sep 10: 449-453, 1998
\end{abstract}

Key words: capillary isotachophoresis; Raman spectroscopy; nucleoside; adenosine; cytidine; guanosine; uridine; adenosine 5'-triphosphate

\section{INTRODUCTION}

Nucleosides and related compounds have major effects on coronary and cerebral circulation [1], neural modulation [2], control of blood flow [3, 4], respiration, and lipolysis [5]. They are closely associated with brain metabolism and are involved in mental activities such as sleeping and dreaming [6]. It has been suggested that these physiological actions are due to the interactions of nucleosides and related compounds with specific receptors, mediated by the changes of interstitial nucleoside concentration [1]. The ratio of adenosine to adenosine $5^{\prime}$-triphosphate (ATP) is useful for probing the role of ATP as both a neurotransmitter acting on postsynaptic neurons and an inhibitor acting on the presynaptic neuron $[7,8]$.

Because of their neurochemical importance, improved techniques for measurement of nucleosides are needed. Ideally, such techniques should involve minimal sample preparation and should provide unambiguous identification of individual nucleosides as

Presented at the Nineteenth International Symposium on Capillary Chromatography and Electrophoresis, Wintergreen, VA, May 1997

Present address: H. Li, Department of Chemistry, Syracuse University, Syracuse, NY 13244

Correspondence to: M.D. Morris

Contract grant sponsor: National Institutes of Health, contract grant number: GM-53766 well as of the related ribonucleotides with which they are often associated. In this communication we report the first steps toward the use of Raman-detected capillary isotachophoresis for this goal.

Capillary isotachophoresis and capillary zone electrophoresis (CZE) are powerful tools for analyzing ionic species. Both, and especially CZE, are widely utilized to separate small biological ions including amino acids, peptides, ribonucleotides, and sugars [9]. However, nucleosides are neutral molecules at physiological $\mathrm{pH}$ and cannot be separated by electrophoresis. Nucleosides can be derivatized by complexation with borate [10]. The resulting complexes can be separated by CZE. With the addition of a fluorophore, laser-induced fluorescence (LIF) has been utilized for detecting derivatized nucleoside-borate complexes at low concentrations [11].

The commonly employed UV absorption and fluorescence detection provide little qualitative information. Mass spectrometry is the most widely employed method for species identification [12-14]. While it is spectacularly informative, mass spectrometry puts constraints on the kinds and ionic strength of buffers employed and on the acceptable range of electro-osmotic flow conditions.

Our research group demonstrated advantages to the use of Raman spectroscopy as a qualitative and even quantitative detection method for capillary electrophoretic separations. We have used normal Raman and resonance Raman to monitor electro- 
phoretic separations of samples ranging from ribonucleotides [15] to herbicides [16] to inorganic oxyanions [17]. Raman spectroscopy requires only simple interfacing and can be used with any buffer. Because analyte Raman spectra disappear into the water spectrum at concentrations near millimolar, it is necessary to preconcentrate samples to obtain Raman spectra. But Raman spectroscopy probes a length of the separation capillary 1-5 $\mu \mathrm{m}$. For this reason capillary isotachophoresis (ITP), which yields concentrated but contiguous zones, can be used effectively with Raman spectroscopic detection. With simple chemometrics high-quality Raman spectra can be reconstructed from the acquired spectra from analytes at starting concentrations below $10^{-6} \mathrm{M}$, or even below $10^{-7} \mathrm{M}$ in favorable cases [16].

During ITP of anions, such as nucleoside-borate complexes, preconcentration of the analyte anions is carried out in a discontinuous buffer system. The analytes are injected between a leading electrolyte which has higher anion mobility than the analytes and a terminating electrolyte with lower anion mobility. Because of the differences in resistance, the lower the mobility of a substance in a given zone, the higher is the electric field strength in that zone. Every ion is constrained to move at the same velocity. The analyte ions migrate as consecutive zones without dilution by the background electrolyte. The concentration of the analyte zones will approach the higher concentration of the leading electrolyte. Stacking of the analytes will occur. Analyte concentrations can easily be increased by $10^{3}-10^{4}$ times.

The Raman spectra of nucleosides and related compounds in aqueous solution are well known. Most have strong Raman bands in the 500-2000$\mathrm{cm}^{-1}$ range [18-21]. Typically, the most intense bands are in-plane motions of the aromatic rings. Sugar vibrations are also observed, but usually with lower intensities.

\section{EXPERIMENTAL}

The ITP apparatus consisted of a $-30-\mathrm{kV}$ high-voltage power supply (Glassman PS/LG-30R-5) which was connected to the injection end of the capillary. The injection end was contained in a Plexiglas safety enclosure. The capillary was held on the microscope stage in a $\mathrm{V}$-groove machined into an aluminum block. The $50-\mu \mathrm{m}$-i.d. fused-silica capillary was alkylsilane derivatized, followed by adsorbing a surfactant. The silylation reagent was octadecyltrichlorosilane and the surfactant was methyl cellulose. The silylation was carried out at $90^{\circ} \mathrm{C}$ in the oven. In most runs the distance from the capillary entrance to the detection window was $23 \mathrm{~cm}$ and the overall length was $38 \mathrm{~cm}$. Samples were injected electrokinetically at $-10 \mathrm{kV}(263 \mathrm{~V} / \mathrm{cm})$ or $-12 \mathrm{kV}(316 \mathrm{~V} / \mathrm{cm})$. In most cases the ITP ran at the same voltage. Loading volumes were $40-250 \mathrm{~nL}$, depending on the injection time used.

The Raman microprobe consisted of a modified Olympus BH-2 epi-fluorescence microscope with 20 $\times / 0.46$ NA microscope objective for illumination and scattered light collection. An 85-mm f/1.8 axial transmissive spectrograph (Holospec, Kaiser Optical Systems, Inc.) fitted with a holographic transmission grating and a cryogenically cooled charge-coupled device (CCD) camera was used. In earlier work a front-illuminated $1024 \times 256$-pixel detector chip (CH-270, Photometrics Ltd.) was employed. This was replaced by a $1340 \times 100$-pixel back-thinned chip (ST-138, Princeton Instruments, Inc.) in later experiments. Spectra were acquired with 0.5 or $1.0 \mathrm{~s}$ integration using CCD9000 software (Photometrics Ltd.) and processed in GRAMS 386 (Galactic Industries) or using Winspec (Princeton Instruments, Inc.) and processed in GRAMS/32 (Galactic Industries). A $50-\mu \mathrm{m}$ spectrograph entrance slit was employed to give a resolution of about $8 \mathrm{~cm}^{-1}$. The exciting laser was a diode-pumped $\mathrm{NdYVO}_{4}$ laser operating at 532 $\mathrm{nm}$ (Spectra Physics) which delivered up to $900 \mathrm{~mW}$ to the capillary. All spectra were calibrated against an argon discharge lamp.

Adenosine, guanosine, cytidine, and uridine were from Aldrich (Milwaukee, WI). Boric acid was from Mallinckrodt (Paris, KY). Tris was from Life Technologies (Gaithersburg, MD). Adenosine 5'-triphosphate, octadecyltrichlorosilane, and methyl cellulose were from Sigma (St. Louis, MO). All common chemicals were of ACS analytical reagent grade. Electrolytes and samples were prepared from Type I deionized water. Nucleoside sample solutions were diluted with $10 \mathrm{mM}$ borate $(\mathrm{pH} 10)$. All solutions contained $0.1 \%$ methyl cellulose used to form the dynamic capillary coating.

\section{RESULTS AND DISCUSSION}

Raman spectra of nucleoside-borate complexes acquired on-capillary are shown in Figure 1. Each sample has been preconcentrated to $10^{-2}-10^{-3} \mathrm{M}$ before reaching the detection window. Comparison of nucleoside-borate complexes and pure nucleoside solution shows that complexation with borate does not change the Raman bands in the 600-1800$\mathrm{cm}^{-1}$ spectral window. The bands are predominately those of the nucleic acid bases, not ribose, and any band shifts should be less than the resolution of our spectrograph. Table I shows the strong Raman bands of the nucleoside-borate complexes and includes the literature assignments for most of them. 


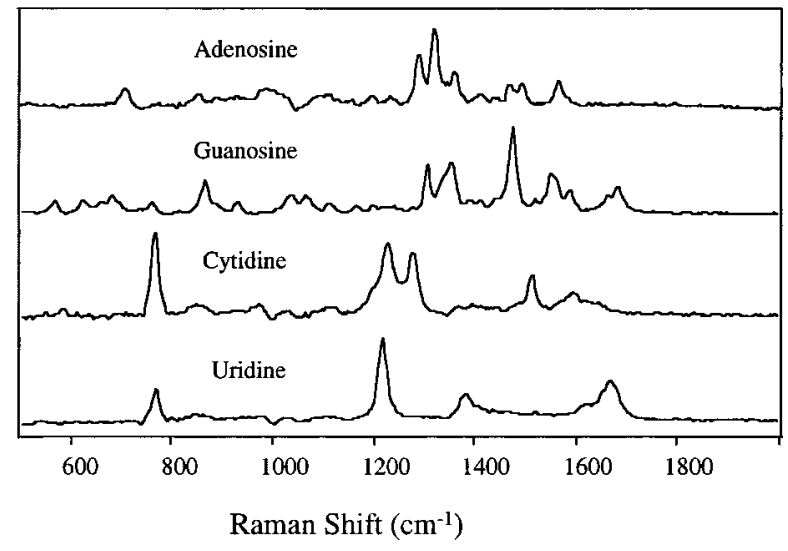

Figure 1. On-capillary Raman spectra of nucleoside-borate complexes preconcentrated to $10^{-2}-10^{-3}$ $M$ by ITP.

Figure 2 shows the separation of the two purine nucleosides, adenosine and guanosine, with starting concentrations of $5 \times 10^{-4} \mathrm{M}$. The injection time was $60 \mathrm{~s}$. The three-dimensional isotachopherogram consists of spectra taken at 58 spectra $/ \mathrm{min}$. With a $-10-\mathrm{kV}$ ITP running voltage, the experiment required only $9 \mathrm{~min}$. The earliest spectra are of the leading electrolyte. The nucleoside zones, which begin at approximately $525 \mathrm{~s}$, are easily identified by their characteristic Raman bands. Guanosine elutes before adenosine, indicating that the guanosineborate complex has a higher effective mobility than the adenosine-borate complex.

There are two fluorescent zones located before and after guanosine. In this display format the fluorescence would obscure the view of anything eluting later. For visual clarity the fluorescent zones are shown in the figure as blank areas. As we have previously demonstrated, an important advantage of ITP/Raman spectroscopy is that ITP functions as an easy sample purification technique which can

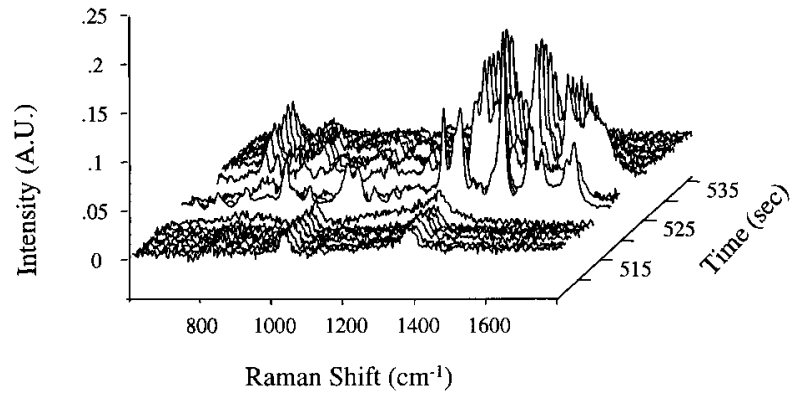

Figure 2. On-capillary Raman isotachopherogram of $5 \times 10^{-4} M$ adenosine and guanosine borate complexes. Injection: $60 \mathrm{~s}$ at $-10 \mathrm{kV}$, running voltage $-10 \mathrm{kV}, 420 \mathrm{~mW}, 532 \mathrm{~nm}$ excitation, $1 \mathrm{~s}$ integration.

effectively eliminate from Raman spectra much of the fluorescence arising from matrix impurities. From the point of view of the Raman spectroscopist spectral interferences are spatiotemporally separated from the materials of interest.

The isotachopherogram for the two pyrimidine nucleosides at starting concentrations of $5.0 \times 10^{-4}$ $M$ is shown in Figure 3. The injection time was $60 \mathrm{~s}$. The uridine-borate complex precedes the cytidine-

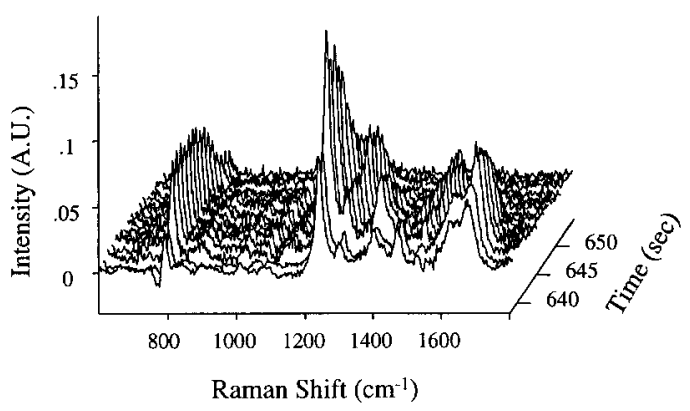

Figure 3. On-capillary Raman isotachopherogram of $5 \times 10^{-4} M$ cytidine and uridine borate complexes; conditions as in Figure 2.

Table I. Assignment of some Raman bands $\left(\mathrm{cm}^{-1}\right)$ of nucleoside-borate complexes.

\begin{tabular}{ccccc}
\hline Adenosine & Guanosine & Cytidine & Uridine & Assignment \\
\hline 735 & 892 & 790 & 792 & ring breathing \\
& 1331 & 1252 & 1242 & $\begin{array}{c}\text { ring stretching } \\
\text { Kekule ring motion }\end{array}$ \\
1317 & 1377 & & & $\begin{array}{c}\text { ribose vibration } \\
1346\end{array}$ \\
1384 & 1497, & $1533(\mathrm{C}=\mathrm{N})$ & 1402 & $\begin{array}{c}\text { purine and pyrimidine ring } \\
\text { double-bond stretching }\end{array}$ \\
1492,1514, & 1571 & & $1685(\mathrm{C}=\mathrm{O})$ & \\
\hline
\end{tabular}




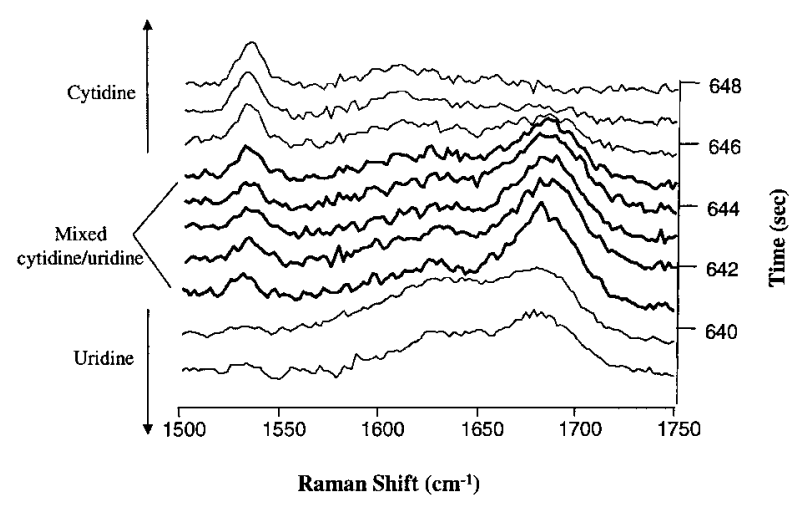

Figure 4. Expanded view of the uridine-cytidine interdiffusion zone; conditions as in Figure 2.

borate complex. While there are no fluorescence problems, there is an interdiffusion region where mixed spectra are seen. The interdiffusion region is more visible in Figure 4. This figure is an expanded view of a 10 -s interval of the isotachopherogram, showing the transition from uridine to cytidine. To emphasize the change, only the $1500-1750-\mathrm{cm}^{-1}$ region is included, where the spectrum of each compound contains only a single band.

The $1533 \mathrm{~cm}^{-1}$ peak is a ring stretch of cytidine, and the $1685 \mathrm{~cm}^{-1}$ band is the $\mathrm{C}=\mathrm{O}$ stretch of uridine. The five spectra from 641 to $645 \mathrm{~s}$ plotted with heavy lines contain mixed uridine and cytidine. Both the 1533 and 1685 bands are present. At $640 \mathrm{~s}$ and earlier the spectra are of uridine only and show only the 1533 band. At $646 \mathrm{~s}$ and later the spectra are of cytidine only, with only the 1685 band.

Separation of adenosine and guanosine at initial concentrations in the micromolar range can be achieved with a longer injection time. Figure 5 shows the separation at a starting concentration of $5.0 \times$

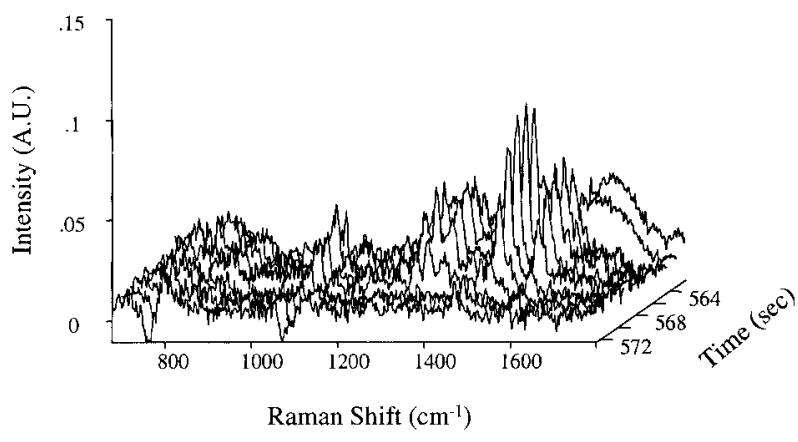

Figure 5. On-capillary Raman spectra of $5 \times 10^{-6}$ $M$ adenosine and guanosine borate complexes. Injection: 5 min at $-10 \mathrm{kV}$, running voltage $-10 \mathrm{kV}, 800$ $\mathrm{mW}, 532 \mathrm{~nm}$ excitation, $0.5 \mathrm{~s}$ integration.
$10^{-6} \mathrm{M}$ in each. The injection time was $5 \mathrm{~min}$, corresponding to an injection volume of about 240 $\mathrm{nL}$. The nucleosides are visible and easily detected. The estimated detection limit is about $1.5 \times 10^{-6}$ $\mathrm{M}$, corresponding to a mass limit of detection of approximately $0.1 \mathrm{ng}$.

Both mass limits of detection and concentration limits of detection can in principle be reduced substantially. A confocal Raman microprobe samples a reaction volume which is approximately coincident with the cylindrical focal volume of the laser. With the $20 \times / 0.46$ objective the volume is about $2 \mu \mathrm{m}$ diameter and $8 \mu \mathrm{m}$ long. Although it will substantially increase the difficulty of alignment and operation, replacement of the $50-\mu \mathrm{m}$-i.d. capillary with a $5-\mu \mathrm{m}$-i.d. capillary would allow more efficient interrogation of the capillary volume. Since detection limits depend upon isotachophoretic stacking conditions, not on capillary dimensions, a $10 \times$ reduction in capillary diameter would yield a $100 \times$ reduction in mass limit of detection.

The separation of a mixture of the four ribonucleosides is shown in Figure 6. Each nucleoside zone is characterized by its Raman fingerprint. The four representative spectra on the right show guanosine, uridine, adenosine, and cytidine, respectively. Curiously, the migration order is purine, and pyrimidine, purine, and pyrimidine. The reasons for this interesting alternation in hydrodynamic radii are not yet understood.

Figure 7 shows the Raman-detected isotachopherogram of a mixture of the borate complexes of adenosine and ATP. The more highly charged ATP elutes first. There is a zone of fluorescence between the two analyte zones which saturates the detector. The adenosine and ATP zones are displayed sepa-

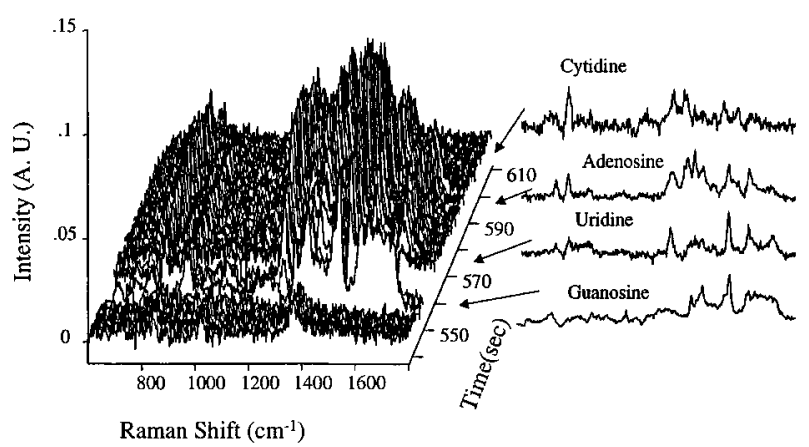

Figure 6. On-capillary Raman isotachopherogram of $5 \times 10^{-4} \mathrm{M}$ adenosine, guanosine, cytidine, and uridine borate complexes. Injection: $4 \mathrm{~min}$ at $-10 \mathrm{kV}$, running voltage $-10 \mathrm{kV}, 420 \mathrm{~mW}, 532 \mathrm{~nm}$ excitation, $1 \mathrm{~s}$ integration. 


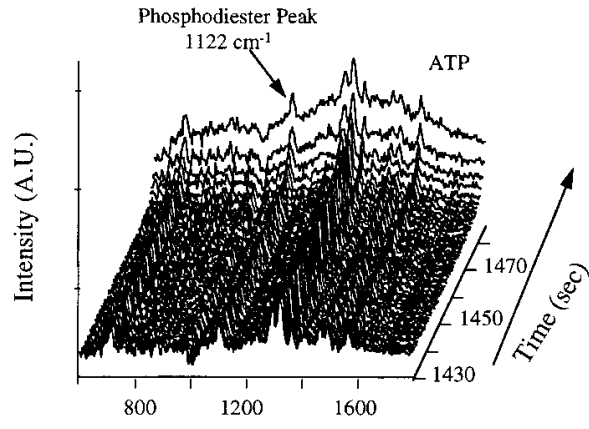

Raman shift $\left(\mathrm{cm}^{-1}\right)$

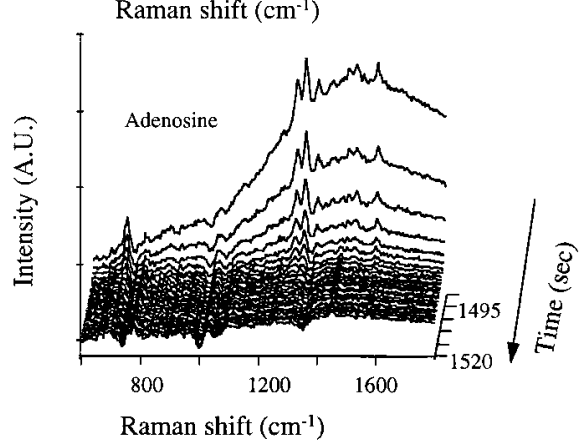

Figure 7. On-capillary Raman isotachopherogram of $5 \times 10^{-4} \mathrm{M}$ adenosine and ATP borate complexes. 50 s injection at $-12 \mathrm{kV}, 700 \mathrm{~mW}, 532 \mathrm{~nm}$ excitation, $1 \mathrm{~s}$ integration. The evolution of the ATP zone (upper traces) is shown from its beginning until the onset of a fluorescence zone. The evolution of the adenosine-borate zone is shown from the end of the fluorescent impurity zone. Note that the time axis has been reversed for visual clarity.

rately to facilitate comparison of the spectra. Because they have similar structures, the two species have similar spectra, except that the borate complex of ATP has a phosphodiester band at $1122 \mathrm{~cm}^{-1}$.

With ITP/Raman spectroscopy, nucleosides can be separated into consecutive, contiguous zones and characterized by their Raman spectra even at starting concentrations approaching $10^{-6} \mathrm{M}$. With optimization and with the application of chemometrics detection and identification of submicromolar concentrations should be feasible. Identification of nucleosides in plasma, tissue, and cell extracts should be possible as well.

\section{ACKNOWLEDGMENT}

We thank Kaiser Optical Systems, Inc. for consignment of the Raman spectrograph. Jeremy Shaver provided much useful advice on the use of GRAMS $/ 32$.

\section{REFERENCES}

1. S.C. Hermann and E.O. Deigl, J. Chromatogr. B 574, 247 (1992).

2. G. Gamberini, V. Ferioli, P. Zanoli, M.L. Zeneroli, C. Rustichelli, and M. Baraldi, Chromatograpia 34, 563 (1992).

3. R.M. Berne, Circ. Res. 47, 807 (1980).

4. R.M. Berne, R.M. Knabb, S.W. Ely, and R. Rubio, Fed. Proc. Fed. Am. Soc. Exp. Biol. 42, 3136 (1983).

5. A. Kurtz, J. Biol. Chem. 262, 6296 (1987).

6. G.L. Stiles, D.T. Daly, and R.A. Olsson, J. Neurochem. 47, 1020 (1986).

7. J.W. Phillis and P.H. Wu, Progr. Neurobiol. 16, 187 (1981).

8. M. Williams, Ann. Rev. Pharmacol. Toxicol. 27, 315 (1987).

9. J.P. Landers, Ed., Handbook of Capillary Electrophoresis (CRC Press, Boca Raton, FL, 1994), Part III.

10. S.P. Atamas and G.V. Troitsky, J. Chromatogr. 644, 407 (1993).

11. C-C Wang, W.P. McCann, and S.C. Beale, J. Chromatogr. B 676, 19 (1996).

12. M.A. Moseley, J.W. Jorgenson, J. Shabanowitz, D.F. Hunt, and K.B. Tomer, J. Am. Soc. Mass. Spectrom. 3, 289 (1992).

13. K.P. Bateman, R.L. White, and P. Thibault, Rapid Commun. Mass Spectrom. 11, 307 (1997).

14. J.F. Kelly, L. Ramaley, and P. Thibault, Anal. Chem. 69, 51 (1997).

15. P.A. Walker III, W.K. Kowalchyk, and M.D. Morris, Anal. Chem. 67, 4255 (1995).

16. P.A. Walker III, J.M. Shaver, and M.D. Morris, Appl. Spectrosc. 51, 1394 (1997).

17. W.K. Kowalchyk, P.A. Walker III, and M.D. Morris, Appl. Spectrosc. 49, 1183 (1995).

18. P. Carmona and M. Molina, J. of Molec. Struct. 219, 323 (1990).

19. J. Goral and V. Zichyt, Spectrochim. Acta 46A(2), 253 (1990).

20. R.C. Lord and G.J. Thomas, Jr., Biochim. Biophys. Acta 142, 1 (1967).

21. S. Tanaka, T. Kaneta, and H. Yoshida, J. Chromatogr. 498, 205 (1990). 